\title{
Tolerancia a la alimentación inmediata en los pacientes con pancreatitis biliar leve
}

\author{
Immediate feeding tolerance in patients with mild acute biliary pancreatitis \\ David Esmer*, Oscar Rivera-Villalobos, Juan F. Hernández-Sierra, Liliana D. Valencia-Sánchez y \\ Martín Sánchez \\ Hospital Central "Ignacio Morones Prieto", Facultad de Medicina, Universidad Autónoma de San Luis Potosí, San Luis Potosí, México
}

\section{Resumen}

Antecedentes: La pancreatitis aguda leve es una inflamación local del páncreas sin complicaciones locales ni falla orgánica. Su manejo consiste en tres elementos básicos: hidratación, analgesia y ayuno. La realimentación se inicia cuando el paciente no tiene dolor y refiere apetito, pero el momento exacto para iniciarla no está previamente documentado. Objetivo: Determinar la tolerancia a la alimentación oral inmediata (8 horas posterior al inicio del manejo) en comparación con la alimentación temprana (48 horas) en los pacientes con pancreatitis aguda biliar leve. Método: Se incluyeron pacientes con pancreatitis aguda biliar leve y se aleatorizaron en dos grupos: A) alimentación temprana a las 48 horas (30 pacientes) y B) alimentación inmediata a las 8 horas de inicio del manejo (29 pacientes). Fueron evaluados por un tercer observador ciego (no involucrado en el estudio) para documentar síntomas como náusea, vómito, reactivación del dolor abdominal, síndrome de respuesta inflamatoria sistémica, tolerancia alimentaria y estancia hospitalaria. Resultados: No se encontraron diferencias significativas entre los grupos en cuanto a datos clínicos y bioquímicos. La estancia hospitalaria disminuyó significativamente en el grupo $B$ (5.4 vs. 7.8 días; $p$ < 0.003). Conclusión: La alimentación inmediata en las primeras 8 horas del manejo de los pacientes con pancreatitis aguda biliar leve es bien tolerada y segura, y reduce la estancia hospitalaria.

Palabras clave: Alimentación. Dolor abdominal. Pancreatitis biliar aguda. Respuesta inflamatoria.

\section{Abstract}

Background: Mild acute pancreatitis is defined as pancreatic edema, without organic failure and without local complications. Its management consists in three basic elements: hydration, analgesia and fasting. Start refeeding when the patient haven't pain and referring hungry, but exact time for this is not previously documented. Objective: To determine the tolerance to immediate oral feeding (8 hours after the start of management) compared to early feeding (48 hours) in patients with mild acute biliary pancreatitis. Method: Included all patient with mild acute biliary pancreatitis, and they were randomized in to two groups: A) early oral feeding $(n=30)$ and B) immediate oral feeding $(n=29)$. They were evaluated by a third blind observer (not involved in the study) for documented symptoms like nausea, vomiting, reactivation of abdominal pain, systemic inflammatory response syndrome, food tolerance and hospital stay. Results: There were no significant differences between both groups in the clinical data or in the biochemical studies. The hospital stay was significantly less in the group $B$ (5.4 vs. 7.8 days; $p<$ 0.003). Conclusion: Immediate oral feeding is well tolerated and secure in patients with mild acute biliary pancreatitis.

Key words: Feeding. Abdominal pain. Acute biliary pancreatitis. Systemic inflammatory response syndrome.

\section{Correspondencia:}

*David Esmer

Palmira, 600-302

Col. Pedregal

Fecha de recepción: 27-11-2019

C.P. 78214, San Luis Potosí, S.L.P., México

E-mail: esmer_david@hotmail.com
Cir Cir. 2021;89(2):243-247

Contents available at PubMed www.cirugiaycirujanos.com

0009-7411/@ 2020 Academia Mexicana de Cirugía. Publicado por Permanyer. Este es un artículo open access bajo la licencia CC BY-NC-ND (http://creativecommons.org/licenses/by-nc-nd/4.0/). 


\section{Introducción}

La pancreatitis aguda leve se define como un edema de la glándula pancreática sin falla orgánica ni complicaciones locales ${ }^{1}$, y su manejo consiste en tres elementos básicos: hidratación, analgesia y reposo intestinal temporal|2-7.

El concepto de reposo intestinal asume una disminución en la inflamación, el dolor y la secreción de enzimas pancreáticas en el parénquima y el tejido peripancreático, principalmente para evitar una reactivación de la pancreatitis. Diversos estudios han mostrado, al comparar pacientes en ayuno contra el inicio temprano de la alimentación oral, que no existen diferencias significativas en la reactivación de la pancreatitis ${ }^{7-11}$. También se ha demostrado que existe una mayor respuesta inmunitaria con la alimentación temprana, la cual reduce los procesos infecciosos (bacteriemia, neumonía e infección de la necrosis en la pancreatitis grave) y la mortalidad general ${ }^{12,13}$.

A pesar de lo mencionado previamente, el tiempo exacto para iniciar la realimentación no se ha definido, por lo que el objetivo del presente trabajo fue evaluar la tolerancia y la reactivación de la enfermedad al iniciar la alimentación oral inmediata (8 horas desde el inicio del manejo hospitalario) en los pacientes con pancreatitis aguda biliar leve (PABL) en comparación con el inicio temprano (48 horas desde el inicio del manejo).

\section{Método}

Estudio controlado, doble ciego y aleatorizado realizado en el Hospital Central Dr. Ignacio Morones Prieto. Se incluyeron pacientes mayores de 15 años, de ambos sexos, con diagnóstico de PABL, clasificados de acuerdo con las escalas de Ranson $<3$, APACHE II $<8$ y BISAP $<2^{1,14,15}$. Todos los pacientes contaron con ultrasonido hepático y de vía biliar para comprobar la etiología. Se excluyeron los pacientes con pancreatitis de etiología no biliar, pancreatitis grave, riesgo alto de coledolitiasis o colangitis. Los pacientes permanecieron cegados a la hipótesis del estudio en todo momento.

Los pacientes fuero aleatorizados en dos grupos (aleatorización simple con tabla de números aleatorios generados por computadora utilizando el programa paquete $R$, en estratos de cuatro): A) realimentación temprana a las 48 horas de inicio del manejo hospitalario, y B) realimentación inmediata a las 8 horas de inicio del manejo hospitalario. El alimento oral que se utilizó para iniciar la vía oral fue una fórmula polimérica estándar (Enterex Diabetic®, Pisa Pharmaceutical), calculado a sus requerimientos basales ( $25 \mathrm{kcal} / \mathrm{kg}$ de peso).

Se determinaron los leucocitos y la proteína $\mathrm{C}$ reactiva al ingreso y a las 8-24 y 48 horas.

Todos los pacientes tuvieron el mismo manejo inicial, con reanimación hídrica parenteral con solución Hartmann a $15 \mathrm{ml} / \mathrm{kg} / \mathrm{h}$ por las primeras 8 horas y después evaluando la velocidad de infusión necesaria para obtener una diuresis de $0.5 \mathrm{ml} / \mathrm{kg} / \mathrm{h}$. Se utilizó un inhibidor de la bomba de protones a dosis de $40 \mathrm{mg} / 24 \mathrm{~h}$ i.v.; como analgesia, metamizol a dosis de $2 \mathrm{~g} / 6 \mathrm{~h}$ i.v. y ketorolaco a dosis de $30 \mathrm{mg} / 8 \mathrm{~h}$ i.v.; y como procinético-antiemético, metoclopramida a dosis de $10 \mathrm{mg} / 8 \mathrm{~h}$ i.v.

En ambos grupos, la tolerancia a la alimentación fue determinada por un observador ciego al grupo del paciente: si el paciente cumplía los criterios de ausencia de dolor, náusea o vomito, y tenía hambre en el momento en que se le ofreció la dieta y además la toleraba sin mostrar datos de respuesta inflamatoria sistémica ni recurrencia del dolor.

A todos los pacientes se les realizó colecistectomía con colangiografía intraoperatoria después de la resolución de la pancreatitis, determinándose como resolución de la enfermedad, al momento en que el paciente toleró la vía oral. Los pacientes se egresaron 24 horas tras la cirugía. Se consideró como estancia hospitalaria el tiempo transcurrido desde el ingreso hasta el egreso.

Se calculó el tamaño de la muestra bajo la asunción de $\mathrm{p} 1=10 \%$ y $\mathrm{p} 2=30 \%$, con un poder del $80 \%$ y un intervalo de confianza del $95 \%$ a dos colas, obteniendo $n=25$ pacientes por grupo 5 .

El análisis estadístico se realizó con el paquete estadístico JMP versión 8 (SAS Institute Inc., Cary, NC, USA), utilizando la prueba t de Student para variables numéricas con distribución normal o la prueba $U$ Mann Whitney en otros casos. Las variables categóricas se analizaron con la prueba de ji al cuadrado o la prueba exacta de Fischer, considerándose significancia estadística una $p<0.05$.

El presente trabajo fue autorizado por el comité de ética del hospital (Registro 07-15), y se obtuvo el consentimiento informado de todos los pacientes para entrar en el estudio.

\section{Resultados}

Se incluyeron 30 pacientes en el grupo A de alimentación temprana y 29 pacientes en el grupo B de 
Tabla 1. Valores basales en los grupos de estudio

\begin{tabular}{|c|c|c|c|}
\hline & Alimentación temprana $(n=30)$ & Alimentación inmediata $(n=29)$ & p \\
\hline Edad (años) & $38(18-89)$ & $37(20-49)$ & 0.946 \\
\hline Sexo (masculino:femenino) & $5: 25$ & $3: 26$ & 0.302 \\
\hline IMC & $26.96 \pm 4.15$ & $26.35 \pm 4.41$ & 0.586 \\
\hline Inicio de síntomas (h) & $44.4(4-144)$ & $31.10(4-96)$ & 0.172 \\
\hline Leucocitos $\left(10^{3} / \mu l\right)$ & $8.9 \pm 4.3$ & $8.3 \pm 3.99$ & 0.547 \\
\hline Amilasa al ingreso $(\mathrm{U} / \mathrm{l})$ & $1357 \pm 689$ & $1523 \pm 587$ & 0.102 \\
\hline Lipasa (U/I) & $945 \pm 358$ & $1024 \pm 315$ & 0.119 \\
\hline EVA inicial & $5.9(2-10)$ & $5.7(0-10)$ & 0.785 \\
\hline Ranson & 1.37 & 1.3 & 0.276 \\
\hline APACHE II & $4.73 \pm 2.59$ & $4.65 \pm 3.16$ & 0.917 \\
\hline BISAP & 0.36 & 0.17 & 0.287 \\
\hline
\end{tabular}

EVA: escala visual análoga de dolor; IMC: índice de masa corporal.

alimentación inmediata. En la tabla 1 se presentan las características basales de cada grupo. Como se observa, no existen diferencias significativas entre los grupos de estudio, considerándolos comparables.

El dolor abdominal evaluado con la escala visual análoga de dolor no presentó diferencias a las 0,8 y 48 horas: $5.9 \pm 2.35,5.7 \pm 2.58$ y $2.2 \pm 2.1$ para el grupo $A$ vs. $5.7 \pm 2.58,2.17 \pm 2.0$ y $0.13 \pm 0.35$ para el grupo $B$.

La cuenta leucocitaria al inicio de la alimentación fue de $10.72 \pm 4.3 \times 10^{3} / \mu$ l en el grupo $A$ y $10.85 \pm$ $3.99 \times 10^{3} / \mu \mathrm{l}$ en el grupo $\mathrm{B}$, sin diferencia significativa $(p=0.905)$. A las 48 horas, los valores fueron de 9.03 $\pm 6.37 \times 10^{3} / \mu \mathrm{l}$ en el grupo $\mathrm{A}$ y de $7.71 \pm 2.42 \times 10^{3} / \mu \mathrm{l}$ en el grupo $B$, sin diferencia significativa $(p=0.143)$.

La proteína $C$ reactiva medida al ingreso fue de $4.24 \pm 5.85 \mathrm{mg} / \mathrm{dll}$ en el grupo $A$ y de $3.52 \pm 4.4 \mathrm{mg} / \mathrm{dl} \mathrm{en}$ el grupo B, y a las 48 horas fue de $3.71 \pm 5.33$ y 2.05 \pm 2.99 , respectivamente, sin diferencias significativas en ambas mediciones. No se registraron casos con respuesta inflamatoria ni falla orgánica durante la estancia en ninguno de los grupos.

Se presentaron ocho pacientes (13.5\%) con falla a la tolerancia de la vía oral: cinco en el grupo A (16\%) y tres en el grupo $B(10.3 \%)$, sin diferencia estadística ( $p=0.4$ ) (Tabla 2).

Encontramos diferencia estadística en la estancia hospitalaria a favor de la alimentación inmediata comparada con la alimentación temprana: 2.39 vs. 3 días ( $p=0.0001)$ y 5.4 vs. 7.8 días, respectivamente $(p<0.0003)$ (Fig. 1).

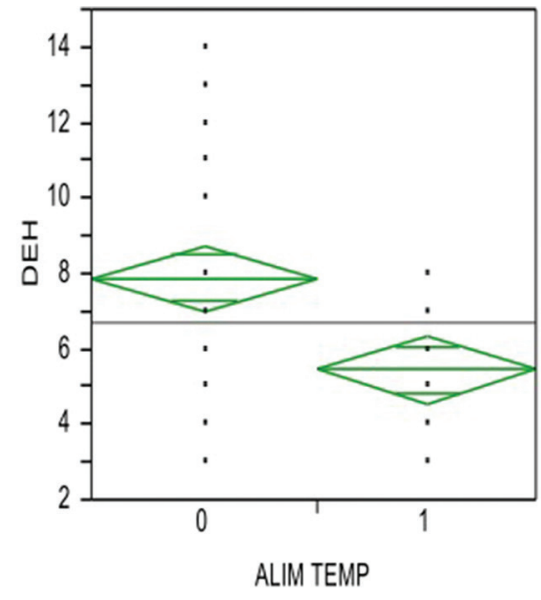

Figura 1. Días de estancia hospitalaria (DEH). Se indican la mediana, el intervalo de confianza del 95\% y los límites.

Se siguió a los pacientes por 30 días y hubo dos readmisiones en el grupo de alimentación temprana, ambas por coledocolitiasis residual, que se resolvieron por colangiopancreatografía retrógrada endoscópica. No documentamos complicaciones locales de la pancreatitis.

\section{Discusión}

El objetivo del ayuno, en el manejo tradicional y fundamental de la pancreatitis aguda, ha sido «el reposo de la glándula pancreática» para evitar la secreción enzimática en respuesta a los estímulos de 
Tabla 2. Pacientes que no toleraron la alimentación

\begin{tabular}{lccccc}
\hline & Alimentación temprana $(\mathbf{n = 3 0})$ & $\%$ & Alimentación inmediata $(\mathbf{n}=\mathbf{2 9})$ & $\%$ & $\mathbf{p}$ \\
\hline Náusea/vómito & 0 & 0 & 0 & 0 & NS \\
Dolor abdominal & 5 & 16.66 & 3 & 10.33 & 0.091 \\
Respuesta inflamatoria sistémica & 0 & 0 & 0 & 0 & NS \\
Falla orgánica & 0 & 0 & 0 & 0 & NS \\
Total & 5 & 16.66 & 3 & 10.33 & 0.091 \\
\hline NS: no significativo. & & & &
\end{tabular}

colecistocinina y minimizar el estrés de la glándula, con el objetivo de disminuir la autodigestión y la exacerbación del daño tisular ${ }^{8}$. Aunque el ayuno inicial está estandarizado y generalmente se tolera bien en los pacientes con pancreatitis aguda leve, el tiempo necesario hasta realimentar al paciente para evitar la reactivación o la exacerbación de la enfermedad ha sido poco estudiado en estudios clínicos aleatorizados. Los estudios actuales sugieren que en la pancreatitis aguda la secreción de zimógenos en respuesta a los alimentos está disminuida, lo que ha permitido evaluar el inicio cada vez más temprano de la realimentación, una vez que la primera fase del tratamiento ha comenzado con hidratación y analgesia, incluso sin una reducción completa de los parámetros inflamatorios, como han mostrado Niederau, et al. ${ }^{16}$ en su estudio.

En este trabajo encontramos grupos homogéneos en sus características basales, sin diferencias en las escalas de gravedad, y todos los pacientes recibieron el mismo tratamiento, por lo que los resultados no pudieron explicarse por desequilibrios en este posible factor de confusión.

En estudios previos, como el de Mendes, et al..$^{11}$, se reporta que la reactivación del dolor durante la alimentación temprana puede ocurrir hasta en el 19\% de los casos, pero en este trabajo solo el $13.5 \%$ de los pacientes presentaron falla de realimentación porque continuaron con dolor abdominal, sin una diferencia estadísticamente significativa, y no tuvimos pacientes a quienes se les reactivó el dolor, lo que sugiere una seguridad en el inicio de la ingesta nutricional en las primeras 8 horas de iniciar el manejo de la pancreatitis.

Los resultados obtenidos con la medición de los reactivos de fase aguda indirectos, como los leucocitos y la proteína $\mathrm{C}$ reactiva, no reportaron diferencias significativas al comparar ambos grupos, en sus mediciones iniciales y a las 48 horas, pero mostraron una tendencia a estar en recuentos más bajos en el grupo de alimentación inmediata. Esto coincide con lo reportado por Petrov, et al. ${ }^{17}$, quienes sugieren que la alimentación temprana desempeña un papel inmunomodulador que atenúa la respuesta inflamatoria en los pacientes con pancreatitis grave $e^{13,17}$.

Solo un número limitado de estudios en pancreatitis han evaluado el impacto de la alimentación enteral en el tejido pancreático. Sahin, et al. ${ }^{18}$ compararon la ingesta oral temprana contra parenteral en la pancreatitis aguda e informaron menos cambios histopatológicos en el tejido pancreático en ratas alimentadas por vía oral, explicado con la hipótesis de que la alimentación oral estimula la producción de hormonas enterales (colecistocinina, motilina y serotonina), y esto produce menores efectos en el proceso inflamatorio del páncreas, así como un efecto trófico en el tejido pancreático, aumentando el flujo sanguíneo pancreático y la motilidad intestinal.

Aun así, no podemos ser categóricos con nuestro estudio al mencionar que la alimentación inmediata acelera el periodo en el que remite la pancreatitis; solo podemos observar que no hay una mayor reactivación del dolor y que no hay efectos nocivos en la recuperación de la enfermedad.

La justificación a favor de la alimentación inmediata nos ha permitido intervenir a los pacientes con PABL en forma más temprana y tener una estancia hospitalaria casi 2 días más corta.

En conclusión, nuestro estudio muestra que la alimentación inmediata establecida a las 8 horas de iniciado el manejo de un paciente con PABL es bien tolerada, es segura, no afecta negativamente la evolución de la enfermedad y permite disminuir la estancia hospitalaria. En cuanto al dolor, se presentó con más frecuencia en la alimentación temprana, por lo 
que los estudios que incluyeran un mayor tamaño de muestra tendrían una probabilidad pequeña de demostrar o invertir el resultado; sin embargo, se recomienda realizar estudios como el presente en diferentes poblaciones de edad y con otros factores, como obesidad y manejo hospitalario.

\section{Conflicto de intereses}

Los autores declaran que para este protocolo de investigación no se tiene algún conflicto de intereses.

\section{Responsabilidades éticas}

Protección de personas y animales. Los autores declaran que los procedimientos seguidos se conformaron a las normas éticas del comité de experimentación humana responsable y de acuerdo con la Asociación Médica Mundial y la Declaración de Helsinki.

Confidencialidad de los datos. Los autores declaran que han seguido los protocolos de su centro de trabajo sobre la publicación de datos de pacientes.

Derecho a la privacidad y consentimiento informado. Los autores han obtenido el consentimiento informado de los pacientes y/o sujetos referidos en el artículo. Este documento obra en poder del autor de correspondencia.

\section{Bibliografía}

1. Banks $P$, Bollen T, Dervenis C, Gooszen HG, Johnson CD, Sarr MG, et al. Classification of acute pancreatitis - 2012: revision of the Atlanta classification and definitions by international consensus. Gut. 2013;62:102-11.
2. Working group IAP/APA. IAP/APA evidence-based guidelines for the management of acute pancreatitis. Pancreatology. 2013;13:e1-15.

3. Tenner S, Baillie J, DeWitt J, Vege SS; American College of Gastroenterology. American College of Gastroenterology guideline: management of acute pancreatitis. Am J Gastroenterol. 2013;218:1-16.

4. Fisher JM, Gardner TB. The "golden hours" of management in acute pancreatitis. Am J Gastroenterol. 2012;107:1146-50.

5. Cebrián JG, Bello-Camara MP, Rodríguez-Tanez JC, Fernandez-Ruiz A Analgesia y sedación en la pancreatitis aguda. Med Intensiva. 2003;27(2):118-30.

6. Jiménez Murillo L, Montero Pérez FJ. Medicina de urgencias y emergencias. Guía diagnóstica y protocolos de actuación. 4를 ed. Barcelona: Elsevier; 2010.

7. Spanier B, Bruno M, Mathus-Vliegen VM. Enteral nutrition and acute pancreatitis: a review. Gastroenterol Res Pract. 2011;2011:857949.

8. Eckerwall GE, Tingstedt BB, Bergenzaun PE, Andersson RG. Immediate oral feeding in patients with mild acute pancreatitis is safe and may accelerate recovery. Clin Nutr. 2007;26:758-63.

9. Teich N, Aghdassi A, Fischer J, Walz B, Caca K, Wallochny T, et al. Optimal timing of oral refeeding in mild acute pancreatitis. Pancreas. 2010;39:1088-92.

10. Li J, Xue GJ, Liu YL, Javed MA, Zhao XL, Wan MH, et al. Early oral refeeding wisdom in patients with mild acute pancreatitis. Pancreas. 2013;42:88-91.

11. Moraes JM, Felga GE, Chebli LA, Franco MB, Gomes CA, Gaburri PD, et al. A full solid diet as the initial meal in mild acute pancreatitis is safe and result in shorter length of hospitalization: results from a prospective, randomized, controlled, double-blind clinical trial. J Clin Gastroenterol. 2010;44:517-22.

12. Bakker OJ, van Santvoort HC, van Brunschot S, Ahmed Ali U, Besselink MG, Boermeester MA, et al. Pancreatitis, very early compared with normal start of enteral feeding (PYTHON trial): design and rationale of randomized controlled multicenter trial. Trials. 2011;12:73.

13. Eatock F, Chong P, Menezes N, Murray L, McKay CJ, Carter CR, et al. $A$ randomized study of early nasogastric versus nasojejunal feeding in severe acute pancreatitis. Am J Gastroenterol. 2005;100:432-9.

14. Papachristou GI, Muddana V, Yadav D, O'Connell M, Sanders MK, Slivka A, et al. Comparison of BISAP, Ranson's, APACHE II, and CTSI Scores in predicting organ failure, complication, and mortality in acute pancreatitis. Am J Gastroenterol. 2010;105:435-41.

15. Maraví-Poma E, Patchen Dellinger E, Forsmark CE, Layer P, Lévy $P$, Shimosegawa $T$, et al. Clasificación internacional y mutidisciplinaria de la pancreatitis aguda: edición española 2013. Med Intensiva. 2014;38:211-7.

16. Niederau C, Niederau M, Luthen R, Strohmeyer G, Ferrell LD, Grendell JH. Pancreatic exocrine secretion in acute experimental pancreatitis. Gastroenterology. 1990;99:1120-7.

17. Petrov M, Windsor J. Nutritional management of acute pancreatitis: the concept of "gut rousing". Curr Opin Clin Metab Care. 2013;16:557-63.

18. Sahin M, Ozer S, Vatansev C, Aköz M, Vatansev H, Aksoy F, et al. The impact of oral feeding on the severity of acute pancreatitis. Am J Surg. 1999;178:394-8. 